Please do not remove this page

RMIT

UNIVERSITY

\title{
A time-frequency approach for the analysis of normal and arrhythmia cardiac signals
}

Mahmoud, Seedahmed; Fang, Qiang; Davidovic, Dragomir; Cosic, Irena

https://researchrepository.rmit.edu.au/esploro/outputs/9921862625101341/filesAndLinks?institution=61RMIT_INST\&index=null

Mahmoud, S., Fang, Q., Davidovic, D., \& Cosic, I. (2006). A time-frequency approach for the analysis of normal and arrhythmia cardiac signals. Proceedings of the 28th Annual International Conference of the IEEE Conference on Engineering in Medicine and Biology Society, 2006, 6509-6512.

https://doi.org/10.1109/IEMBS.2006.260882

Published Version: https://doi.org/10.1109/IEMBS.2006.260882

Repository homepage: https://researchrepository.rmit.edu.au

(c) 2006 IEEE. Personal use of this material is permitted. However, permission to reprint/republish this material for advertising or promotional purposes or for creating new collective works for resale or redistribution to servers or lists, or to reuse any copyrighted component of this work in other works must be obtained from the IEEE.

Downloaded On 2023/04/26 22:55:33 +1000 


\title{
A Time-Frequency Approach for the Analysis of Normal and Arrhythmia Cardiac Signals
}

\author{
Seedahmed S. Mahmoud, Qiang Fang, Dragomir M. Davidović and Irena Cosic
}

\begin{abstract}
Previously, electrocardiogram (ECG) signals have been analyzed in either a time-indexed or spectral form. The reality, is that the ECG and all other biological signals belong to the family of multicomponent nonstationary signals. Due to this reason, the use of time-frequency analysis can be unavoidable for these signals. The Husimi and Wigner distributions are normally used in quantum mechanics for phase space representations of the wavefunction. In this paper, we introduce the Husimi distribution (HD) to analyze the normal and abnormal ECG signals in time-frequency domain. The abnormal cardiac signal was taken from a patient with supraventricular arrhythmia. Simulation results show that the HD has a good performance in the analysis of the ECG signals comparing with the Wigner-Ville distribution (WVD).
\end{abstract}

\section{INTRODUCTION}

Conventionally, electrocardiogram (ECG) signal is analyzed in the time-domain by experienced physicians. However, pathological conditions may not always be obvious in the time-domain signal [1]. This fact has motivated the use of frequency domain techniques, such as Fourier transform (FT), for analysis [2]. However, as the ECG and all other biological signals belong to the family of multicomponent nonstationary signals [3], accurate time-varying spectral estimates can be extremely difficult to obtain. However, a proper time-frequency distribution (TFD) can deal with this problem and reveal the multicomponent nature of such signals. The time-frequency presentation provides information where the time-domain and frequency-domain may fail to produce. In particular, it uses to detect the QRS complex and arrhythmia [4].

The Wigner-Ville distribution (WVD) was first defined by E. Wigner in the context of quantum mechanics [5], and later independently introduced by J. Ville for signal processing and spectral analysis [6]. Though Wigner-Ville gives high resolution in time-frequency domain, it is not used widely for practical application due to the interaction between different signal components, the so called "cross term". The Wigner function cannot be directly interpreted as a probability distribution function because, in the general case, it is necessarily negative in some regions of phase space. For an indirect probabilistic interpretation, a non-negative phase space function is necessary. The phase space distribution which is produced in simultaneous unsharp measurements of position and momentum, can be represented as a convolution

S. S. Mahmoud, Q. Fang, and I. Cosic are with the School of Electrical and Computer Engineering, RMIT University, Melbourne, Victoria 3000, Australia mahmoud.seedahmed@rmit.edu.au, john.fang@rmit.edu.au,irena.cosic@rmit.edu.au.

Davidović is with the Vinca Institute of Nuclear Sciences, Laboratory for Theoretical Physics, Yugoslavia davidd@vin.bg.ac.yu . of the Wigner function of considered quantum state and the Wigner function of the filter state which represents a measuring apparatus. If the Wigner function of the filter state is coherent state, in which coordinate and momentum are defined by a minimum uncertainty, what corresponds to optimal unsharp measurement allowed by the Heisenberg uncertainty principle, the corresponding phase space function is Husimi function. In this case the mentioned convolution of Wigner function of a quantum state and a filter state reduces to the absolute square of the scalar product of wave function of a considered state and the coherent state centered at the point $(q, p)$ of phase space for which the Husimi function is calculated. Both Wigner and Husimi functions are the phase space distribution in quantum mechanics. Husimi function is the first function to be introduced as a non-negative distribution function [7]. In this paper, we introduce this distribution, HD, to the analysis of biomedical signals and mapping the phase space representations into time-frequency representations.

\section{ACQUISITION OF NORMAL AND ARRHYTHMIA CARDIAC SIGNALS}

In this study we consider two types of biological signals. These signals are:

1) abnormal cardiac signals, ECG, for a patient with supraventricular arrhythmia (this arrhythmia data was obtained from the Physionet database [8]) and,

2) normal ECG signal (Lead-I).

\section{A. Abnormal Electrocardiogram}

The ECG signal has a well-defined P, QRS, T signature that represents with each heart beat. The P-wave arises from the depolarization of the atrium. The QRS complex arises from depolarization of the ventricles and T-wave arises from repolarization of the ventricle muscles. The duration, shape and amplitude of these waves are considered as major features in time domain analysis. Sometimes, the time morphologies of these waves are similar. An arrhythmia is an abnormality in the heart's rhythm, or heart beat pattern. The heart beat can be too slow, too fast, have extra beats, or otherwise beat irregularly [9], [10]. Supraventricular arrhythmia occurs in the upper areas of the heart and is less serious than ventricular arrhythmia. It has irregular shapes of QRS complexes [9].

A length of 3.9 seconds of the signal-800 from supraventricular arrhythmia database was converted into digital forms by Matlab and processed by the TFD. The sampling frequency for this signal is 128 samples/second. The QRS 
complexes in this signal are wide and abnormal, while the T-wave has disappeared. A TFD with high resolution is vital and crucial in this sort of signal [11].

\section{B. Normal ECG Signal}

In this measurement, Lead-I electrodes was connected to BIOPAC systems, ECG module. The ECG module (ECG 100C) consists of instrumentation amplifier (IA) and a $50 \mathrm{~Hz}$ notch filter. The IA gain was set to 1000 . The ECG data were recorded by using AcqKnowledge software (v.3.7.1, BIOPAC Systems, Inc., CA) in ASCII text files and processed by programs written in Matlab. The sampling rate for ECG was set to 200 samples/second.

\section{Husimi AND Wigner-Ville Distributions}

A TFD that provides a good reduction of the cross-terms is needed to make sure that the off-diagonal elements of the TFD matrix of the sources are negligible and so that a diagonal structure can be maintained. The continuous timefrequency distribution of the analytic signal $z(t)$ associated with the original real signal $s(t)$ can be expressed as follows [12], [13]

$$
\rho(t, f)=\underset{\tau \rightarrow f}{\mathscr{F}}\left[G(t, \tau) \underset{t}{*} K_{z}(t, \tau)\right]
$$

where $K_{z}(t, \tau)=z(t+\tau / 2) z^{*}(t-\tau / 2)$ is the instantaneous autocorrelation product, $\mathscr{F}$ is the Fourier transform, $G(t, \tau)$ is the time-lag kernel, and $*_{t}$ denotes time convolution. It is well-known that the kernel can completely characterize the TFD and its properties (e.g., resolution) [13].

In this study we introduce the Husimi distribution to timefrequency analysis of biological signals and compare its performance with WVD.

\section{A. The Wigner-Ville distribution}

The Wigner - Ville distribution (WVD) of a continuous signal $z(t)$ is defined as [14]

$$
W V(t, f)=\int_{-\infty}^{\infty} z\left(t+\frac{\tau}{2}\right) z^{*}\left(t-\frac{\tau}{2}\right) e^{-j 2 \pi f \tau} d \tau
$$

where $f$ is the frequency variable. The WVD satisfies a large number of desirable mathematical properties. In particular, WVD is always real-valued, it preserves time and frequency shifts and satisfies the marginal properties. Based on (1), the WVD, which utilizes a time-only kernel $G(t, \tau)=\mathrm{G}(t)=$ $\delta(t)$ with $g(v, \tau)=\mathrm{g}(v)=1$, has significant oscillatory cross-terms without a controlling factor, where the crossterms can be larger in amplitude than the auto-terms.

\section{B. Husimi Distribution}

Wigner and Husimi functions are the phase space distribution in quantum mechanics. They provide a two-dimensional picture of a one-dimension wave-function, and can be compared directly with classical phase space distributions [15]. The Husimi distribution function can be given in terms of WVD as follows

$$
H(t, f)=\int_{-\infty}^{\infty} \int_{-\infty}^{\infty} W V(t-u, f-v) e^{-a u^{2}-b v^{2}} d u d v
$$

where $a=\frac{1}{\Delta u}, b=\frac{1}{\Delta v}, W V(t, f)$ is Wigner-Ville distribution and $\Delta u . \Delta v \leq \frac{1}{2 \pi}$. Eq. (3) can be re-written in the following form

$$
H(t, f)=\int_{-\infty}^{\infty} \int_{-\infty}^{\infty} W V(t-u, f-v) x(u) h(v) d u d v .
$$

We can further reduce eq. (4) to the following form

$$
\begin{aligned}
H(t, f) & =x(t) \underset{t}{*} \underset{f}{[}[h(f) * W V(t, f)] \\
& =\gamma(t, f)_{t f}^{* * W V}(t, f)
\end{aligned}
$$

where $\gamma(t, f)=\underset{\tau \rightarrow f}{\mathscr{F}}[G(t, \tau)]$. Based on the general formula of Cohen's class in (1) we can get the kernel for Husimi distribution as follows

$$
G(t, \tau)=e^{-a t^{2}} \sqrt{\frac{\pi}{b}} e^{\frac{-\pi^{2}}{b} \tau^{2}}
$$

where $\tau$ is the time lag. The resolution and cross-terms reduction of Husimi function depends on the values of " $a$ " and " $b$ " in (6).

\section{RESULTS AND DISCUSSION: A COMPARISON OVER NORMAL AND ABNORMAL CARDIAC SIGNALS}

In this section, we compare the performance of the proposed distribution with WVD over normal and abnormal cardiac signals.

\section{A. A comparison over Abnormal ECG of a Patient with Supraventricular Arrhythmia}

A length of 3.9 seconds of the signal-800 from supraventricular arrhythmia database was converted by Hilbert transform into its analytical forms and processed using the proposed distribution, HD, and the WVD. This signal has normal QRS complexes duration of 0.1 second width and a short P-R interval. The QRS complexes have irregular shapes.

Figs. (1) and (2) show the time and 3D time-frequency representations of this signal utilizing HD and WVD, respectively. The proposed distribution manages to detect the three QRS complexes in the signal with their exact shape, however, the WVD fails to track changes in the frequency components of the QRS complex of this signal due to crossterms. All frequency components in this signal are clearly revealed by the proposed distribution, HD.

\section{B. A comparison over Normal ECG Signal}

A normal ECG signal of length 4.9 seconds was converted by Hilbert transform into its analytical forms and processed using the HD and the WVD. Figs. (3) and (4) show the time and 3D time-frequency representations of this signal, normal ECG, utilizing HD and WVD, respectively. Also from these figures, we observe that the HD has best resolution, as it can reveal the frequency components of the normal ECG signal with a resolution much higher than that given by WVD. The 


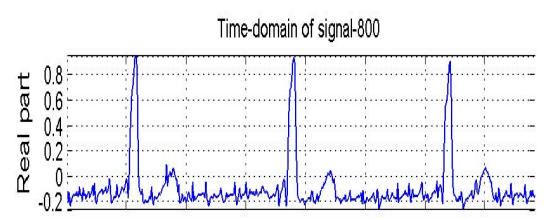

HD, lin. scale, mesh, Threshold $=5 \%$

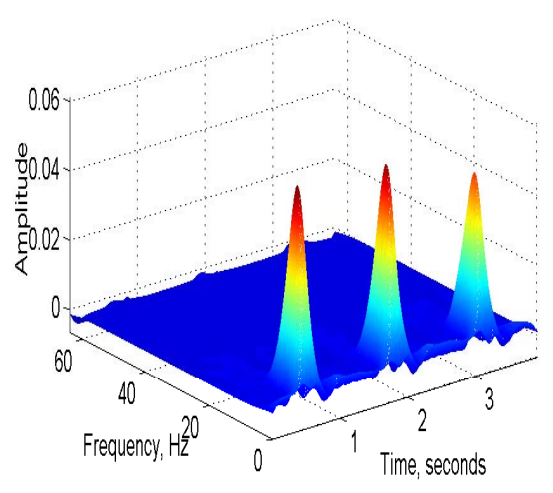

Fig. 1. Time and 3D time-frequency representations of the supraventricular arrhythmia signal using HD. The sampling frequency was $f_{s}=128$ samples/second. For HD, $a=0.02$ and $b=$ 1000 .

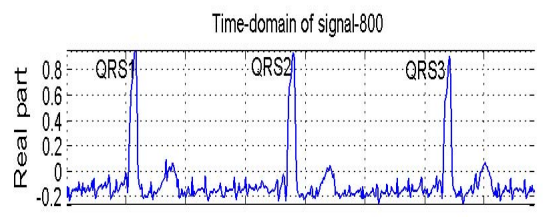

WW, lin. scale, mesh, Threshold =5\%

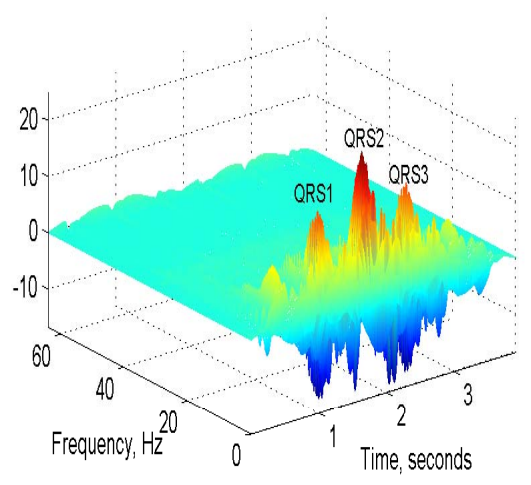

Fig. 2. Time and 3D time-frequency representations of the supraventricular arrhythmia signal using WVD. The sampling frequency was $f_{s}=128$ samples/second.

cross-terms in WVD make the task of identifying the QRS peaks and T-wave extremely difficult.

\section{CONCLUSIONS}

This paper introduced the Husimi distribution (HD) to biomedical signal processing and in particular to time-
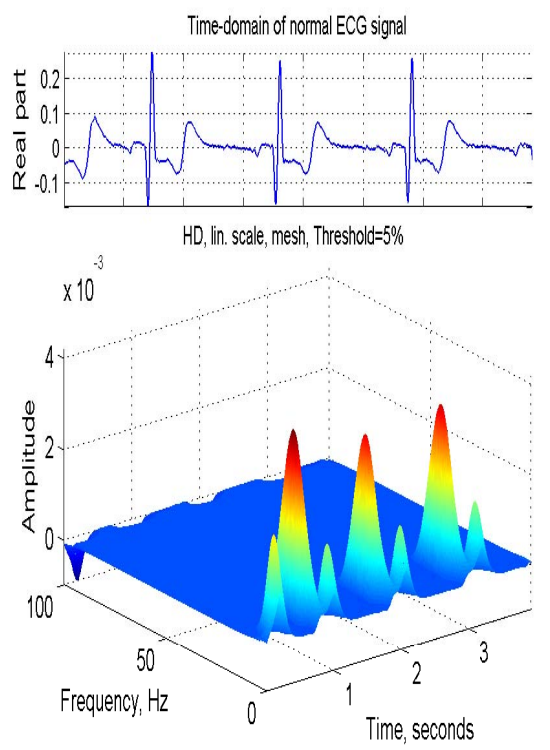

Fig. 3. Time and 3D time-frequency representations of the normal ECG signal using HD. The sampling frequency was $f_{s}=200$ samples/second. For HD, $a=0.02$ and $b=1000$.
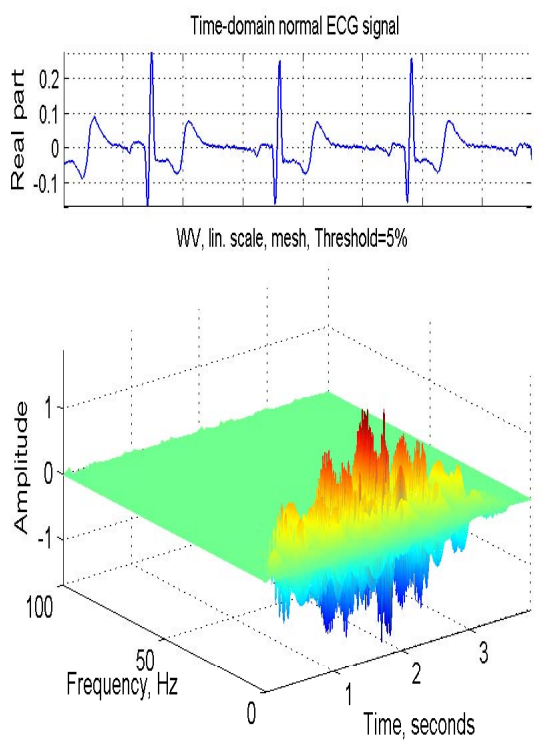

Fig. 4. Time and 3D time-frequency representations of the normal ECG signal using WVD. The sampling frequency was $f_{s}=200$ samples/second.

frequency representation of biological signals. Husimi distribution is a popularly function used in quantum physics. The performance of HD over normal and abnormal cardiac signals was compared with that of the WVD. In case of normal and abnormal signals (a patient with supraventricular arrhythmia), the HD outperforms the WVD in terms of timefrequency resolution in this analysis. The high resolution of 
the HD in revealing the location in the joint time - frequency plane of the QRS complexes is useful in cardiac abnormality detection. The cross terms in the WVD make the task of identifying the QRS peaks and their actual shape extremely difficult, where the cross terms in some figures are larger in amplitude than the auto-terms.

\section{ACKNOWLEDGMENTS}

This research is supported by a RIF grant from RMIT University, Australia.

\section{REFERENCES}

[1] C. Bigan and M. S. Woolfson, Time-frequency analysis of short segments of biomedical data, IEEE Proc.-Sci. Meas. Technol., vol. 147, no. 6, pp. 368-373, Nov. 2000.

[2] R.H. Clayton, A. Murray, "Estimation of the ECG signal spectrum during ventricular fibrillation using the fast Fourier transform and maximum entropy methods," Proceedings of the Computers in Cardiology, pp. 867 - 870, Sept. 1993.

[3] J. C. Wood, D. T. Barry, Time-frequency analysis of skeletal muscle and cardiac vibrations, Proceedings of the IEEE, vol. 84, issue 9, pp. 1281-1294, Sept. 1996.

[4] L. Qiu and G. Li, "Representation of ECG Signals Based on The Instantaneous Frequency Estimation," Proceedings of ICSP, pp. 17311734, 1996.

[5] E. P. Wigner, On the Quantum Correction For Thermodynamic Equilibrium, Phys. Rev., 40-749, 1932.

[6] J. Ville, Theorie et applications de la notion de signal analitique, Cables et Transmission, 2A:61-74, 1948.

[7] K. Husimi, Proc. Phys. Math. Soc. Japan. 22, 264, 1940.

[8] Physiobank, Physionet, Physiologic signal archives for biomedical research, http://www.physionet.org/physiobank/, viewed Aug. 2005.

[9] A. K. Hebbar, and W. J. Hueston, Management of Common Arrhythmias: Part I. Supraventricular Arrhythmias, Journal of American Family Physician, vol. 65, no. 12, pp. 2479-2486, June 2002.

[10] A. K. Hebbar, and W. J. Hueston, Management of Common Arrhythmias: Part II. Ventricular Arrhythmias and Arrhythmias in Special Populations, Journal of American Family Physician, vol. 65, no. 12, pp. 2491-2496, June 2002.
[11] Seedahmed S. Mahmoud, Zahir M. Hussain, Irena Cosic, and Qiang Fang, Time-Frequency Analysis of Normal and Abnormal Biological Signals, Journal of Biomedical Signal Processing and Control, Elsevier, in press.

[12] L. Cohen, Time-Frequency Analysis, Prentice Hall PTR, Englewood Cliffs, New Jersey, 1995.

[13] Z. M. Hussain and B. Boashash, "Adaptive instantaneous frequency estimation of multi-component FM signals using quadratic timefrequency distributions," IEEE Transactions on Signal Processing, vol. 50, no. 8, pp. $1866-1876$, Aug. 2002.

[14] LJ. Stankovic, V. Katkovnic, "The Wigner distribution of noisy signals with adaptive time-frequency varying window," IEEE Trans. Signal Processing, vol. 47, no. 4, pp. 1099-1108, 1996.

[15] D. M. Davidović and D. Lalović, When does a given function in phase space belong to the class of Husimi distributions?, J. Phys. A: Math. Gen. 26, pp. 5099-5105, 1993. 